TAIWANESE JOURNAL OF MATHEMATICS

Vol. 16, No. 2, pp. 429-444, April 2012

This paper is available online at http://journal.taiwanmathsoc.org.tw

\title{
EXISTENCE RESULTS FOR VECTOR SADDLE POINTS PROBLEMS
}

\author{
O. Chadli and H. Mahdioui
}

\begin{abstract}
In this paper, we study the existence of solutions for vector saddle points problems in a general setting. Our approach, first, is based on the KKM lemma and a relaxation of the $C$ - lower semicontinuity notion introduced by T.Tanaka by means of an extension to the vector setting of a Brezis-NirenbergStampacchia condition, and arguments from generalized convexity. This leads us to generalize and improve some new existence results on vector saddle points problems. In the second approach, we establish an existence result for vector saddle point problems under a paracompacity assumption.
\end{abstract}

\section{INTRODUCTION}

Let $X$ and $Y$ be two Hausdorff topological vector spaces, $Z$ be an ordered topological vector space with order associated to a closed convex cone $C$ such that int $C \neq \emptyset$ and $C \neq Z$ where int $C$ denotes the interior of $C$. Note that $C \neq Z$ if and only if int $C$ does not contain the zero vector.

We consider the following vector saddle-point problem : find a pair $\left(u_{0}, v_{0}\right) \in$ $U \times V$ such that

$$
(V S P)\left\{\begin{array}{lc}
f\left(u, v_{0}\right)-f\left(u_{0}, v_{0}\right) \notin-i n t C & \forall u \in U \text { and } \\
f\left(u_{0}, v_{0}\right)-f\left(u_{0}, v\right) \notin-i n t C & \forall v \in V
\end{array}\right.
$$

where $U$ and $V$ are two nonempty subsets, not necessarily compacts, of two real topological vector spaces and $f$ is a vector-valued function from $U \times V$ to $Z$.

Note that when $C=\mathbb{R}^{+}$, problem $(V S P)$ is reduced to the problem of finding a saddle point of a real-valued function, i.e. find $\left(u_{0}, v_{0}\right) \in U \times V$ such that

$$
f\left(u, v_{0}\right) \leq f\left(u_{0}, v_{0}\right) \leq f\left(u_{0}, v\right) \text { for all } u \in U \text { and } v \in V .
$$

Many studies of vector-valued optimization problems seem to lead, in a natural way, to the investigation of vector-saddle point problems in a more general setting, see for

Received October 12, 2010, accepted November 30, 2010.

Communicated by Jen-Chih Yao.

2010 Mathematics Subject Classification: 90C47, 90C48, 49J35,

Key words and phrases: Vector saddle point, C-lower semicontinuity, C-quasiconvexity, KKM lemma, Paracompacity. 
instance $[1,2]$. Problem $(V S P)$ has been investigated by many researchers, see [36]. More precisely, in [6], the authors used a scalarization procedure (see Luc [7]) and some extension to the vector setting of arguments from generalized convexity. Recently in [8], problem $(S V P)$ has been considered by an approach based on a maximal point theorem and some $C$-continuity and $C$-convexity notions related to the cone $C$.

In this paper and in a first approach, we study problem $(V S P)$ by a procedure based on the Fan-KKM Theorem [23], a finite intersection theorem, a relaxation of the $C$-lower (upper) semicontinuity notion introduced by T. Tanaka [11] and arguments from generalized convexity. More precisely, we introduce a condition which in fact represents an extension to the vector setting of the Brezis-NirenbergStampacchia condition [15]. This leads us to relax the $C$-lower (upper) semicontinuity notion due to T. Tanaka. In a second approach, we study problem $(V S P)$ in a setting of paracompact spaces.

The paper is organized as follows. In section 2 , we give some preliminary notions and results that we will need in the sequel, and we state some definitions and properties concerned with lower semicontinuity and convexity for vector-valued functions. In section 3, we show an existence theorem for vector-valued saddlepoint problem by an approach as described above. Section 4 is devoted to vector saddle point problems under a paracompacity assumption.

\section{Preliminaries}

In this section, we give some definitions and preliminary results that we will need in the sequel. For a given set $A$ of $X$, we shall denote by $\operatorname{co}(A)$ the convex hull of $A, \operatorname{int}(A)$ the interior of $A$ and $\bar{A}$ the closure of $A$.

Definition 2.1. ([12]). Let $X$ be a topological space, $K$ a nonempty convex subset of $X$ and $Z$ be an ordered topological vector space with order associated to a closed convex cone $C$ such that int $C \neq \emptyset$ and $C \neq Z$. A vector-valued function $f: K \rightarrow Z$ is said to be $C$-properly quasiconvex on $K$ if

$$
f\left(t u_{1}+(1-t) u_{2}\right) \in f\left(u_{1}\right)-C
$$

or

$$
f\left(t u_{1}+(1-t) u_{2}\right) \in f\left(u_{2}\right)-C
$$

for all $u_{1}, u_{2} \in K$ and $t \in[0,1]$.

Remark 2.1. A vector-valued function $f: K \rightarrow Z$ is said to be $C$-properly quasiconcave if $(-f)$ is $C$-properly quasiconvex.

In the following lemma, we give a useful property that we will need in the sequel. 
Lemma 2.1. Let $U$ and $V$ be two nonempty convex subsets of two topological vector spaces $X$ and $Y$ respectively, let $f: U \times V \rightarrow Z$ be a vector-valued function $C$-properly quasiconvex with respect to the first argument, let $y \in V$ and $E$ be nonempty finite subset of $U$. For all $z \in Z$, if $f(\bar{x}, y)-z \in(-i n t C)^{c}$ for some $\bar{x} \in \operatorname{co}(E)$, then there exists $x \in E$ such that $f(x, y)-z \in(-i n t C)^{c}$.

Proof. Let $E=\left\{x_{1}, \ldots, x_{n}\right\}$ be finite subset of $U$ and

$$
\bar{x}=\sum_{i=1}^{n} \lambda_{i} x_{i}, \quad \text { where } \lambda_{i} \geq 0 \text { for all } i \text { with } \sum_{i=1}^{n} \lambda_{i}=1 .
$$

Let $z \in Z$. If $n=2$, by assumption, for all $y \in V$, the vector-valued function $f(\cdot, y)$ is $C$-properly quasiconvex on $U$, we have

$$
f\left(\lambda_{1} x_{1}+\lambda_{2} x_{2}, y\right)-z \in f\left(x_{1}, y\right)-z-C
$$

or

$$
f\left(\lambda_{1} x_{1}+\lambda_{2} x_{2}, y\right)-z \in f\left(x_{2}, y\right)-z-C .
$$

If $f\left(x_{1}, y\right)-z \in-i n t C$ and $f\left(x_{2}, y\right)-z \in-i n t C$, then we are led to the contradiction

$$
f\left(\lambda_{1} x_{1}+\lambda_{2} x_{2}, y\right)-z \in\left\{f\left(x_{1}, y\right), f\left(x_{2}, y\right)\right\}-z-C \in-i n t C .
$$

Hence $f\left(x_{1}, y\right)-z \in(-i n t C)^{c}$ or $f\left(x_{2}, y\right)-z \in(-i n t C)^{c}$.

Now, we shall complete the proof by applying mathematical induction to $n$. Let $z \in Z$ and assume that $f\left(\sum_{i=1}^{n+1} \lambda_{i} x_{i}, y\right)-z \in(-i n t C)^{c}$. Let $\lambda=\sum_{i=1}^{n} \lambda_{i}=1-\lambda_{n+1}$ and $x=\sum_{i=1}^{n} \frac{\lambda_{i}}{\lambda} x_{i}$. Since $\bar{x}=\lambda x+\lambda_{n+1} x_{n+1}$, then $f(x, y)-z \in(-i n t C)^{c}$ or $f\left(x_{n+1}, y\right)-z \in(-i n t C)^{c}$. If $f(x, y)-z \in(-i n t C)^{c}$, then by the induction hypothesis $f\left(x_{i}, y\right)-z \in(-i n t C)^{c}$ for some $i$, which completes the proof.

Definition 2.2. ([11]). Let $X$ be a topological space. A vector-valued function $f: X \rightarrow Z$ is said to be $C$-lower semicontinuous on $X$ if it satisfies one of the following three equivalent conditions:

(i) For each $u_{0} \in X$ and any $d \in \operatorname{int}(C)$, there exists an open neighborhood $U$ of $u_{0}$ such that

$$
f(u) \in f\left(u_{0}\right)-d+\operatorname{int}(C), \forall u \in U .
$$

(ii) For all $z \in Z, f^{-1}(z+\operatorname{int}(C))$ is open.

(iii) For each $u_{0} \in X$ and any open neighborhood $V$ of $f\left(u_{0}\right)$, there exists an open neighborhood $U$ of $u_{0}$ such that

$$
f(u) \in V+C, \forall u \in U .
$$




\section{Remark 2.2.}

1. When $Z=\mathbb{R}$ and $C=\mathbb{R}^{+}$, the $C$-upper semicontinuity notion is the same as ordinary upper semicontinuity.

2. Clearly, a vector-valued function $f$ is $C$-upper semicontinuous if and only $-f$ is C-lower semicontinuous, and vice versa. It is obvious that every continuous mapping is $C$-lower semicontinuous and $C$-upper semicontinuous. Conversely, if $f$ is $C$-lower semicontinuous and $C$-upper semicontinuous simultaneously, then it is continuous whenever $C$ has a closed convex bounded base; see Theorem 5.3 on p. 22 of [7].

The next proposition represents a characterization of $C$-upper (lower) semicontinuity in terms of generalized sequences, see [19].

Proposition 2.1. Let $X$ be a Hausdorff topological space, and let $f$ be a vectorvalued function from $X$ to $Z$. Then $f$ is $C$-upper semicontinuous on $X$ if and only if for every $x \in X$, for every $v \in$ int $C$, and for any generalized sequence $\left\{x_{\alpha}\right\}_{\alpha \in I}$ in $X$ converging to $x$, there is an $\alpha_{0}$ in the index set I such that

$$
\overline{\left\{f\left(x_{\beta}\right): \beta \succeq \alpha\right\}} \subset f(x)+d-i n t C
$$

for all $\alpha \succeq \alpha_{0}$.

Proof. Suppose that $f$ is $C$-upper semicontinuous. For $\alpha \in I$, let $A_{\alpha}=$ $\left\{f\left(x_{\beta}\right): \beta \succeq \alpha\right\}$. Since $f$ is $C$-upper semicontinuous, then there exists an open neighborhood $U$ of $x$ such that $f(y) \in f(x)+\frac{1}{2} v-i n t(C)$ for all $y \in U$. There is an $\alpha_{0}$ in the index set $I$ such that

$$
\alpha \succeq \alpha_{0} \Longrightarrow x_{\alpha} \in U \text { and } f\left(x_{\alpha}\right) \in f(x)+\frac{1}{2} v-\operatorname{int}(C) .
$$

This implies that $A_{\alpha} \subset f(x)+\frac{1}{2} v-\operatorname{int}(C)$ and $\overline{A_{\alpha}} \subset f(x)+\frac{1}{2} v-C$ whenever $\alpha \succeq \alpha_{0}$. Since $\frac{1}{2} v-C=v-\frac{1}{2} v-C \subset v-\operatorname{int}(C)$, then

$$
\overline{A_{\alpha}} \subset f(x)+v-\operatorname{int}(C) \text { for all } \alpha \succeq \alpha_{0} .
$$

Conversely, assume that $f$ is not $C$-upper semicontinuous on $X$. Then there is a $z_{0} \in Z$ such that $f^{-1}\left(z_{0}-\operatorname{int}(C)\right)$ is not open in $X$. Hence there is an $x_{0} \in$ $f^{-1}\left(z_{0}-i n t(C)\right)$ such that every neighborhood of $x_{0}$ is not contained in $f^{-1}\left(z_{0}-\right.$ $\operatorname{int}(C))$. Write $f\left(x_{0}\right)=z_{0}-v_{0}$ for some $v_{0} \in \operatorname{int}(C)$. Then, there exists a generalized sequence $\left\{x_{\alpha}\right\}_{\alpha \in I}$ in $X$ such that $x_{\alpha} \rightarrow x$ and every $f\left(x_{\alpha}\right)$ does not lie in $z_{0}-\operatorname{int}(C)=f\left(x_{0}\right)+v_{0}-i n t(C)$. Since the complement of $f\left(x_{0}\right)+v_{0}-i n t(C)$ is closed, then for every $\alpha \in I$,

$$
\overline{A_{\alpha}} \cap\left(f\left(x_{0}\right)+v_{0}-\operatorname{int}(C)\right)=\emptyset,
$$

which is a contradiction. The proof is complete.

The following proposition is important for our later discussion. 
Proposition 2.2. Let $X$ and $Y$ be two Hausdorff topological vector spaces, let $U \subset X$ and $V \subset Y$ be two nonempty subsets, and let $f: U \times V \rightarrow Z$ be a vector-valued function. Assume that, for all $u \in U$ and $v \in V, f(\cdot, v)$ is $C$-lower semicontinuous on $U$ and $f(u, \cdot) C$-upper semicontinuous on $V$. If for $(u, v) \in U \times V$ and for any generalized sequence $\left\{\left(u_{\alpha}, v_{\alpha}\right)\right\}_{\alpha \in I}$ in $U \times V$ converging to $\left(u_{0}, v_{0}\right)$ satisfy

$$
\begin{gathered}
f\left(t u+(1-t) u_{0}, v_{0}\right)-f\left(u_{\alpha}, v_{0}\right) \notin-i n t C \text { and } \\
f\left(u_{0}, v_{\alpha}\right)-f\left(u_{0}, t v+(1-t) v_{0}\right) \notin-i n t C, \\
\text { for all } t \in[0,1],
\end{gathered}
$$

then

$$
f\left(u, v_{0}\right)-f\left(u_{0}, v_{0}\right) \in(-\operatorname{int} C)^{c} \quad \text { and } f\left(u_{0}, v_{0}\right)-f\left(u_{0}, v\right) \in(-i n t C)^{c} .
$$

Proof. By assumption, we have

$$
\begin{gathered}
f\left(u, v_{0}\right)-f\left(u_{\alpha}, v_{0}\right) \notin-i n t C \text { and } \\
f\left(u_{0}, v_{\alpha}\right)-f\left(u_{0}, v\right) \notin-i n t C .
\end{gathered}
$$

Set $-d=f\left(u, v_{0}\right)-f\left(u_{0}, v_{0}\right)$ and suppose, by contradiction, that $d \in i n t C$. Since the vector-valued function $-f\left(\cdot, v_{0}\right)$ is $C$-upper semicontinuous at the point $u_{0}$, there is an open neighborhood $U_{u_{0}}$ of $u_{0}$ such that $-f\left(w, v_{0}\right) \in-f\left(u_{0}, v_{0}\right)+d-$ intC for all $w \in U_{u_{0}}$. On the other hand, since $\left\{u_{\alpha}\right\}_{\alpha \in I}$ converges to $u_{0}$, there is an $\alpha_{0}$ in the index set $I$ such that

$$
\alpha \succeq \alpha_{0} \Rightarrow u_{\alpha} \in U_{u_{0}} \quad \text { and }-f\left(u_{\alpha}, v_{0}\right) \in-f\left(u_{0}, v_{0}\right)+d-i n t C .
$$

Consequently, $f\left(u, v_{0}\right)-f\left(u_{\alpha}, v_{0}\right) \in-i n t C$ for all $\alpha \succeq \alpha_{0}$ and $u_{\alpha} \in U_{u_{0}}$. This contradicts our assumption. Similarly, we can show that $f\left(u_{0}, v_{0}\right)-f\left(u_{0}, v\right) \in$ $(-i n t C)^{c}$, for all $u \in U$.

\section{Existence Results for Vector Saddle Points: AN INTERSECTION PROPERTY APPROACH}

In this section, we derive existence results for the vector-saddle point problem $(V S P)$. First we recall the well known Fan-KKM Theorem stated below.

Definition 3.1. (KKM-mapping) Let $U$ be a nonempty subset of a topological vector space $X$ and $F: U \rightarrow 2^{X}$ be a set-valued mapping where $2^{X}$ denotes the family of all subsets of $X$. We say that $F$ is a KKM mapping (or the family of sets $\{F(x)\}_{x \in U}$ satisfies the KKM principle) if if for any nonempty finite set $A \subset U$ one has

$$
\operatorname{co}(A) \subset \bigcup_{x \in A} F(x) .
$$


Theorem 3.1. (Fan-KKM Theorem [14]) Let $U$ be a nonempty subset of a topological vector space $X$, and let $F: U \rightarrow 2^{X}$ be a KKM-mapping. If $F(x)$ is closed in $X$ for every $x$, and if $F\left(x_{0}\right)$ is compact for some $x_{0} \in U$, then $\bigcap_{x \in U} F(x)$ is nonempty.

Now we state the main result of this section.

Theorem 3.2. Let $U$ and $V$ be two nonempty convex subsets of two Hausdorff topological vector spaces $X$ and $Y$ respectively and let $f: U \times V \rightarrow Z$ be a vector-valued function satisfying the following conditions:

(i) for every $v \in V$, the function $u \mapsto f(u, v)$ is $C$-properly quasiconvex and $C$-lower semicontinuous on the convex hull of every nonempty finite subset of $U$;

(ii) for every $u \in U$, the function $v \mapsto f(u, v)$ is $C$-properly quasiconcave and $C$-upper semicontinuous on the convex hull of every nonempty finite subset of $V$;

(iii) for an arbitrary $(u, v) \in U \times V$ and for any generalized sequence $\left\{\left(u_{\alpha}, v_{\alpha}\right)\right\}_{\alpha \in I}$ in $U \times V$ converging to $\left(u_{0}, v_{0}\right)$, if

$$
\begin{gathered}
f\left(t u+(1-t) u_{0}, t v+(1-t) v_{0}\right)-f\left(u_{\alpha}, t v+(1-t) v_{0}\right) \notin-i n t C \quad \text { and } \\
f\left(t u+(1-t) u_{0}, v_{\alpha}\right)-f\left(t u+(1-t) u_{0}, t v+(1-t) v_{0}\right) \notin-i n t C \\
\text { for all } \alpha \in I \text { and for } 0 \leq t \leq 1,
\end{gathered}
$$

then

$$
\begin{gathered}
f\left(u, v_{0}\right)-f\left(u_{0}, v_{0}\right) \notin-i n t C \quad \text { and } \\
f\left(u_{0}, v_{0}\right)-f\left(u_{0}, v\right) \notin-i n t C ;
\end{gathered}
$$

(iv) (Coercivity) there is a nonempty compact set $N \times M \subset U \times V$, and there is a non-empty compact convex set $\widetilde{N} \times \widetilde{M} \subset U \times V$ such that if $(x, y) \in$ $(N \times M)^{c} \cap(U \times V)$, then $f(u, \widetilde{v})-f(\widetilde{u}, \widetilde{v}) \in-i n t C$ and $f(\widetilde{u}, \widetilde{v})-f(\widetilde{u}, v) \in$ -intC for some $(\widetilde{u}, \widetilde{v}) \in \widetilde{N} \times \widetilde{M}$.

Then there exists $(\bar{u}, \bar{v}) \in U \times V$ such that

$$
\begin{aligned}
& f(u, \bar{v})-f(\bar{u}, \bar{v}) \in(-i n t C)^{c} \quad \text { for all } u \in U \text { and } \\
& f(\bar{u}, \bar{v})-f(\bar{u}, v) \in(-i n t C)^{c} \quad \text { for all } v \in V .
\end{aligned}
$$

To prove this theorem, we need the following lemma.

Lemma 3.1. Let $X$ and $Y$ be two Hausdorff topological vector spaces, $U \subset X$ and $V \subset Y$ be two nonempty convex subsets, and $f: U \times V \rightarrow Z$ be a vectorvalued function satisfying the following conditions: 
(i) for every $v \in V$, the function $x \mapsto f(x, v)$ is $C$-properly quasiconvex and $C$-lower semicontinuous on the convex hull of every finite subset of $U$;

(ii) for every $u \in U$, the function $y \mapsto f(u, y)$ is $C$-properly quasiconcave and $C$-upper semicontinuous on the convex hull of every finite subset of $V$.

Then for each finite subset $E$ of $U$ and each finite subset $F$ of $V$, there exist $x_{0} \in \operatorname{co}(E)$ and $y_{0} \in \operatorname{co}(F)$ such that

$$
\begin{aligned}
& f\left(u, y_{0}\right)-f\left(x_{0}, y_{0}\right) \in(-i n t C)^{c} \quad \forall u \in c o(E) \text { and } \\
& f\left(x_{0}, y_{0}\right)-f\left(x_{0}, v\right) \in(-i n t C)^{c} \quad \forall v \in c o(F) .
\end{aligned}
$$

Proof. Set $K:=U \times V$ and for $(x, y) \in K$, consider the following subsets

$$
\begin{array}{ll}
T(x, y)=\{u \in U: & \left.f(x, y)-f(u, y) \in(-i n t C)^{c}\right\} \\
L(x, y)=\{v \in V: & \left.f(x, v)-f(x, y) \in(-i n t C)^{c}\right\} .
\end{array}
$$

It is obvious that $x \in T(x, y)$ and $y \in L(x, y)$ for $(x, y) \in K$, therefore the set $T(x, y) \times L(x, y)$ is nonempty. Now, let $E$ and $F$ be two finite subsets of $U$ and $V$, respectively. For $(x, y) \in c o(E \times F)$ consider the following subset

$$
W(x, y)=\{(u, v) \in c o(E \times F): \quad u \in T(x, y) \quad \text { and } \quad v \in L(x, y)\} .
$$

We show that the family of sets $\{W(x, y)\}_{(x, y) \in c o(E \times F)}$ satisfies the KKM principle. To this aim, we proceed as the following:

(a) First, we show that $W(x, y)$ is closed for each $(x, y) \in c o(E \times F)$. Indeed, let $\left\{\left(u_{\alpha}, v_{\alpha}\right)\right\}_{\alpha \in I}$ be a generalized sequence of elements of $W(x, y)$ such that $u_{\alpha} \rightarrow$ $\bar{u} \in \operatorname{co}(E)$ and $v_{\alpha} \rightarrow \bar{v} \in c o(F)$. Suppose on the contrary that $(\bar{u}, \bar{v})$ is not in $W(x, y)$, then $\bar{u} \notin T(x, y)$ or $\bar{v} \notin L(x, y)$. Suppose that $\bar{u} \notin T(x, y)$, then by setting $f(x, y)-f(\bar{u}, y)=-d$, one has $d \in i n t C$. Since the vector-valued function $f(\cdot, y)$ is $C$-lower semicontinuous in $\bar{u}$, there is an open neighborhood $U_{\bar{u}}$ of $\bar{u}$ such that $\forall d^{\prime} \in i n t C$ there is an $\alpha_{0}$ in the index set $I$ such that:

$$
\alpha \geq \alpha_{0} \quad \Rightarrow \quad u_{\alpha} \in U_{\bar{u}} \quad \text { and } \quad-f\left(u_{\alpha}, y\right) \in-f(\bar{u}, y)+d^{\prime}-i n t C .
$$

For $d^{\prime}=d$, one has

$$
f(x, y)-f\left(u_{\alpha}, y\right) \in-i n t C,
$$

which contradicts the fact that $u_{\alpha} \in T(x, y)$. Similarly we prove that $\bar{v} \notin L(x, y)$ also leads to a contradiction. Therefore $(\bar{u}, \bar{v}) \in W(x, y)$.

(b) Note that $\operatorname{co}(E \times F)$ is compact since $X \times Y$ is a Hausdorff space. Since the set $W(x, y)$ is closed in $c o(E \times F)$, it is compact. 
(c) It remains to show that if $\left\{x_{1}, \ldots, x_{n}\right\} \subset \operatorname{co}(E)$ and $\left\{y_{1}, \ldots, y_{m}\right\} \subset \operatorname{co}(F)$, then

$$
\operatorname{co}\left(\left\{x_{1}, \ldots, x_{n}\right\} \times\left\{y_{1}, \ldots, y_{m}\right\}\right) \subset \bigcup_{i=1, j=1}^{n, m} W\left(x_{i}, y_{j}\right) .
$$

Suppose, on the contrary, there exists a finite set $\left\{x_{1}, \ldots, x_{n}\right\} \times\left\{y_{1}, \ldots, y_{m}\right\} \subset$ $c o(E \times F)$ such that

$$
\operatorname{co}\left(\left\{x_{1}, \ldots, x_{n}\right\} \times\left\{y_{1}, \ldots, y_{m}\right\}\right) \not \subset \bigcup_{i=1, j=1}^{n, m} W\left(x_{i}, y_{j}\right) .
$$

Then, there exist $u=\sum_{i=1}^{n} \lambda_{i} x_{i} \in \operatorname{co}\left(\left\{x_{1}, \ldots, x_{n}\right\}\right.$ and $v=\sum_{j=1}^{m} \beta_{j} y_{j} \in \operatorname{co}\left(\left\{y_{1}\right.\right.$, $\left.\left.\ldots, y_{m}\right\}\right)$ such that $u \notin T\left(x_{i}, y_{j}\right)$ or $v \notin L\left(x_{i}, y_{j}\right)$ for $i=1,2, \ldots, n$ and $j=$ $1,2, \ldots, m$. Consider the case where $u \notin T\left(x_{i}, y_{j}\right)$ for $i=1,2, \ldots, n$ and $j=$ $1,2, \ldots, m$. Let $j \in\{1, \cdots, m\}$ be fixed. Since $u \in T\left(u, y_{j}\right)$, we have

$$
f\left(u, y_{j}\right)-f\left(u, y_{j}\right) \notin-i n t C .
$$

Since $f(\cdot, y)$ is $C$-properly quasiconvex, by Lemma 2.1 there exists $x_{i} \in\left\{x_{1}, \ldots, x_{n}\right\}$ such that $f\left(x_{i}, y_{j}\right)-f\left(u, y_{j}\right) \notin-i n t C$, which contradicts the fact that $u \notin$ $T\left(x_{i}, y_{j}\right)$. Similarly, we obtain a contradiction when considering the case $v \notin$ $L\left(x_{i}, y_{j}\right)$ for $i=1,2, \ldots, n$ and $j=1,2, \ldots, m$. This implies that $W$ is a KKMmapping.

Therefore, by the Fan-KKM Theorem one has

$$
\bigcap_{(x, y) \in \operatorname{co}(E \times F)} W(x, y) \neq \emptyset .
$$

Let $\left(u_{0}, v_{0}\right) \in \cap_{(x, y) \in \operatorname{co}(E \times F)} W(x, y)$, then we have

$$
u_{0} \in T(x, y) \quad \text { and } v_{0} \in L(x, y) \text { for all }(x, y) \in \operatorname{co}(E \times F) .
$$

We complete the proof by considering $y=v_{0}$ and $x=u_{0}$ in relation (1).

Proof of Theorem 3.2. First we consider the case where $K:=U \times V$ is a compact set, i.e. $U$ and $V$ are compact subsets of $X$ and $Y$ respectively. Let $\mathfrak{F}$ be the family of all nonempty finite subsets of $K$. For $E \times F \in \mathfrak{F}$, consider the following set

$\mathcal{M}_{E \times F}=\{(u, v) \in K: u \in T(x, y)$ and $v \in L(x, y)$, for all $(x, y) \in c o(E \times F)\}$.

According to Lemma 3.1, $\mathcal{M}_{E \times F}$ is nonempty for every $E \times F \in \mathfrak{F}$. We shall prove that

$$
\bigcap_{E \times F \in \mathfrak{F}} \overline{\mathcal{M}}_{E \times F} \neq \emptyset
$$


Since $K$ is compact, it suffices to show that the family $\mathfrak{M}=\left\{\overline{\mathcal{M}}_{E \times F}: E \times F \in \mathfrak{F}\right\}$ has the finite intersection property. To this end, let $E_{1} \times F_{1}, E_{2} \times F_{2} \in \mathfrak{F}$, and let $E \times F=\left(E_{1} \times F_{1}\right) \cup\left(E_{2} \times F_{2}\right)$. By definition of the set $\mathcal{M}_{E \times F}$, one can easily see that

$\mathcal{M}_{E \times F} \subset \mathcal{M}_{E_{1} \times F_{1}} \cap \mathcal{M}_{E_{2} \times F_{2}}$ and therefore $\emptyset \neq \overline{\mathcal{M}}_{E \times F} \subset \overline{\mathcal{M}}_{E_{1} \times F_{1}} \cap \overline{\mathcal{M}}_{E_{2} \times F_{2}}$.

This proves that the family $\mathfrak{M}$ has the finite intersection property.

Now, let $\left(u_{0}, v_{0}\right) \in \bigcap_{E \times F \in \mathfrak{F}} \overline{\mathcal{M}}_{E \times F}$. For an arbitrary $(u, v) \in K$, let $\widehat{Y}=$ $\left\{\left(u_{0}, v_{0}\right),(u, v)\right\}$. Since $\left(u_{0}, v_{0}\right) \in \overline{\mathcal{M}}_{\widehat{Y}}$, then there exists a generalized sequence $\left\{\left(u_{\alpha}, v_{\alpha}\right)\right\}_{\alpha \in I}$ in $\mathcal{M}_{\widehat{Y}}$ such that $u_{\alpha} \rightarrow u_{0}$ and $v_{\alpha} \rightarrow v_{0}$.

By the definition of $\mathcal{M}_{\widehat{Y}}$, we have

$$
\begin{gathered}
u_{\alpha} \in T\left(t u+(1-t) u_{0}, t v+(1-t) v_{0}\right) \text { and } \\
v_{\alpha} \in L\left(t u+(1-t) u_{0}, t v+(1-t) v_{0}\right), \\
\text { for all } \alpha \in I \text { and for } 0 \leq t \leq 1
\end{gathered}
$$

Consequently, for all $\alpha \in I$, we have

$$
\begin{gathered}
f\left(t u+(1-t) u_{0}, t v+(1-t) v_{0}\right)-f\left(u_{\alpha}, t v+(1-t) v_{0}\right) \notin-i n t C \text { and } \\
f\left(t u+(1-t) u_{0}, v_{\alpha}\right)-f\left(t u+(1-t) u_{0}, t v+(1-t) v_{0}\right) \notin-i n t C \\
\text { for all } \alpha \in I \text { and for } 0 \leq t \leq 1
\end{gathered}
$$

By conditions (iii),

$$
\begin{gathered}
f\left(u, v_{0}\right)-f\left(u_{0}, v_{0}\right) \notin-i n t C \text { and } \\
f\left(u_{0}, v_{0}\right)-f\left(u_{0}, v\right) \notin-i n t C .
\end{gathered}
$$

Now, we consider the general case where $U$ and $V$ are not necessarily compact subsets of $X$ and $Y$ respectively. For every $E \times F \in \mathfrak{F}$, consider the following set

$$
\begin{aligned}
& \mathcal{M}_{E \times F}=\{(u, v) \in N \times M: u \in T(x, y) \\
&\text { and } v \in L(x, y) \text { for all }(x, y) \in \operatorname{co}((E \cup \widetilde{N}) \times(F \cup \widetilde{M}))\} .
\end{aligned}
$$

Note that $\operatorname{co}((E \cup \widetilde{N}) \times(F \cup \widetilde{M}))$ is compact for every $E \times F \in \mathfrak{F}$. Therefore, by what has been proved, one deduces that there exists $(\widetilde{u}, \widetilde{v}) \in \operatorname{co}((E \cup \widetilde{N}) \times(F \cup \widetilde{M}))$ such that

$$
\begin{gathered}
f(u, \widetilde{v})-f(\widetilde{u}, \widetilde{v}) \notin-i n t C \text { for all } u \in \operatorname{co}(E \cup \widetilde{N}) \text { and } \\
f(\widetilde{u}, \widetilde{v})-f(\widetilde{u}, v) \notin-i n t C \text { for all } v \in \operatorname{co}(F \cup \widetilde{M}) .
\end{gathered}
$$


Since $\widetilde{N} \times \widetilde{M} \subset \operatorname{co}((E \cup \widetilde{N}) \times(F \cup \widetilde{M}))$, by the coercivity assumption $(v i)$, one deduce that $(\widetilde{x}, \widetilde{y}) \in N \times M$. This proves that $\mathcal{M}_{E \times F} \neq \emptyset$ for every $E \times F \in \mathfrak{F}$. By the same argument as above, one can easily see that the family $\mathfrak{M}=\left\{\overline{\mathcal{M}}_{E \times F}\right.$ : $E \times F \in \mathfrak{F}\}$ has the finite intersection property. Hence, by the compactness of $N \times M$, one deduce that $\bigcap_{E \times F \in \mathfrak{F}} \overline{\mathcal{M}}_{E \times F} \neq \emptyset$.

Let $\left(u_{0}, v_{0}\right) \in \bigcap_{E \times F \in \mathfrak{F}} \overline{\mathcal{M}}_{E \times F}$. For an arbitrary $(u, v) \in K$, let $\widehat{Y}=\left\{\left(u_{0}, v_{0}\right),(u, v)\right\}$. Since $\left(u_{0}, v_{0}\right) \in \bar{M}_{\widehat{Y}}$, there exists a generalized sequence $\left\{\left(u_{\alpha}, v_{\alpha}\right)\right\}_{\alpha \in I}$ in $\mathcal{M}_{\widehat{Y}}$ such that $\left(u_{\alpha}, v_{\alpha}\right) \rightarrow\left(u_{0}, v_{0}\right)$. By the definition of $\mathcal{M}_{\widehat{Y}}$, we have

$$
\begin{gathered}
u_{\alpha} \in T\left(t u+(1-t) u_{0}, t v+(1-t) v_{0}\right) \\
v_{\alpha} \in L\left(t u+(1-t) u_{0}, t v+(1-t) v_{0}\right) \\
\text { for all } \alpha \in I \text { and for } 0 \leq t \leq 1 .
\end{gathered}
$$

Consequently, for all $\alpha \in I$, we have

$$
\begin{gathered}
f\left(t u+(1-t) u_{0}, t v+(1-t) v_{0}\right)-f\left(u_{\alpha}, t v+(1-t) v_{0}\right) \notin-i n t C \\
f\left(t u+(1-t) u_{0}, v_{\alpha}\right)-f\left(t u+(1-t) u_{0}, t v+(1-t) v_{0}\right) \notin-i n t C \\
\text { for all } \alpha \in I \text { and for } 0 \leq t \leq 1
\end{gathered}
$$

By conditions (iii), we conclude

$$
\begin{gathered}
f\left(u, v_{0}\right)-f\left(u_{0}, v_{0}\right) \notin-i n t C \text { and } \\
f\left(u_{0}, v_{0}\right)-f\left(u_{0}, v\right) \notin-i n t C,
\end{gathered}
$$

which completes the proof.

As a consequence, by taking $Z=\mathbb{R}$ and $C=\mathbb{R}^{+}$, one obtains the following result on the existence of a saddle-point for a real-valued function.

Corollary 3.1. Let $U$ and $V$ be two nonempty and convex subsets of two Hausdorff topological vector spaces $X$ and $Y$ respectively and let $f: U \times V \rightarrow \mathbb{R}$ be a real-valued function satisfying the following conditions:

(i) For every $v \in V$, the function $u \mapsto f(u, v)$ is quasi convex and lower semicontinuous on the convex hull of every nonempty finite subset of $U$.

(ii) For every $u \in U$, the function $v \mapsto f(u, v)$ is quasi concave and upper semicontinuous on the convex hull of every nonempty finite subset of $V$.

(iii) For an arbitrary $(u, v) \in U \times V$ and for any generalized sequence $\left\{\left(u_{\alpha}, v_{\alpha}\right)\right\}_{\alpha \in I}$ in $U \times V$ converging to $\left(u_{0}, v_{0}\right)$, if

$$
\begin{gathered}
f\left(u_{\alpha}, t v+(1-t) v_{0}\right) \leq f\left(t u+(1-t) u_{0}, t v+(1-t) v_{0}\right) \leq f\left(t u+(1-t) u_{0}, v_{\alpha}\right) \\
\text { for all } \alpha \in I \text { and for } 0 \leq t \leq 1,
\end{gathered}
$$


then

$$
f\left(u_{0}, v\right) \leq f\left(u_{0}, v_{0}\right) \leq f\left(u, v_{0}\right) .
$$

(iv) (Coercivity) There is a nonempty compact set $N \times M \subset U \times V$, and there is a non-empty compact convex set $\widetilde{N} \times \widetilde{M} \subset U \times V$ such that if $(x, y) \in$ $(N \times M)^{c} \cap(U \times V)$, then $f(u, \widetilde{v})-f(\widetilde{u}, \widetilde{v})<0$ and $f(\widetilde{u}, \widetilde{v})-f(\widetilde{u}, v)<0$ for some $(\widetilde{u}, \widetilde{v}) \in \widetilde{N} \times \widetilde{M}$.

Then there exists $(\bar{u}, \bar{v}) \in U \times V$ such that

$$
f(\bar{u}, v) \leq f(\bar{u}, \bar{v}) \leq f(u, \bar{v}) \text { for all }(u, v) \in(U \times V) .
$$

Remark 3.3. Theorem 3.2 generalizes the results obtained by Kimura-Tanaka [6], Kimura [8], and notably the ones obtained by Ferro [3] and Kazmi-Khan [4]. We point out that, in the scalar setting, condition $(i v)$ has been considered first by Brézis-Nirenberg-Stampacchia [15] as a relaxation of the pseudomonotonicity notion introduced by Brézis (see Theorem 1, condition 4 in [15]). More precisely as mentioned in Application 3 p. 297 in [15], pseudomonotonicity implies condition 4 in [15, Theorem1]. In our approach and according to Proposition 2.2, condition (iv) represents in fact a relaxation of the $C$-lower (upper) semicontinuity notion introduced by Tanaka [11]. We mention also that the results presented here are in a general setting of Hausdorff topological vector spaces.

\section{Existence Result for Vector-SAddle Point Problems in Paracompact SPACES}

Let $X$ and $Y$ be two Hausdorff topological vector spaces. We denote by $(X \times$ $Y)^{*}$ the topological dual of $X \times Y$. Let $U$ and $V$ are two nonempty convex subsets of $X$ and $Y$ respectively and let $Z$ be an ordered topological vector space with order associated to a closed convex cone $C$ such that $\operatorname{int} C \neq \emptyset$ and $C \neq Z$. Let $f$ be a vector-valued function from $U \times V$ to $Z$.

We consider the following vector saddle-point problem: find a pair $\left(u_{0}, v_{0}\right) \in$ $U \times V$ such that

$$
(V S P)\left\{\begin{array}{l}
f\left(u, v_{0}\right)-f\left(u_{0}, v_{0}\right) \notin-i n t C \quad \forall u \in U \text { and } \\
f\left(u_{0}, v_{0}\right)-f\left(u_{0}, v\right) \notin-i n t C \quad \forall v \in V .
\end{array}\right.
$$

Definition 4.1. A Hausdorff space is paracompact if every open cover of the space has an open locally finite cover, i.e., if $X=\cup_{i \in I} V_{i}$ with $\left\{V_{i}\right\}_{i \in I}$ a family of open subsets of $X$, then there exists $\left\{W_{\alpha}\right\}_{\alpha \in J}$ a family of open sets of $X$ such that for all $\alpha \in J$, there exists $i \in I$ with $W_{\alpha} \subset V_{i}, X=\cup_{\alpha \in J} W_{\alpha}$ and $\forall x \in X$, there exists a neighborhood $U$ of $x$ such that $U$ meets at most finitely many $W_{\alpha}$. 
Remark 4.1. (a) Every compact space is paracompact and every metrizable space is paracompact; see [22, p.68].

(b) Every closed subset of a paracompact space is paracompact.

Definition 4.2. A family $\left\{\beta_{i}\right\}_{i \in I}$ of continuous functions defined from $X$ into $[0,+\infty)$ is called a partition of unity associated to an open cover $\left\{V_{i}\right\}_{i \in I}$ of $X$, if

(i) $\operatorname{Supp}\left(\beta_{i}\right) \subset V_{i}$ for all $i \in I$;

(ii) the family $\left\{\operatorname{Supp}\left(\beta_{i}\right)\right\}_{i \in I}$ is locally finite;

(iii) $\sum_{i \in I} \beta_{i}(x)=1$, for each $x \in X$.

In the following, we recall a condition that characterizes the paracompact sets (see, [22, p. 68]).

Theorem 4.1. A Hausdorff space $X$ is paracompact if and only if every open cover of $X$ has a continuous locally finite partition of unity.

We shall use the following result, see Theorem 10 in [24].

Theorem 4.2. [24]. Let $X$ be a Hausdorff topological vector space, $K$ a closed convex subset of $X$ and $g: K \times K \rightarrow \mathbb{R}$ be a real bifunction satisfying $g(x, x)=0$ for each $x \in K$. Suppose that

(i) for each finite subset $E$ of $K$ one has

$$
\min _{x \in \operatorname{co}(E)} \max _{y \in E} g(x, y) \geq 0
$$

(ii) for each $y \in K$, the function $x \in K \mapsto g(x, y)$ is upper semicontinuous;

(iii) there exists a nonempty compact subset $A$ of $K$, and there is a nonempty compact convex subset $B$ of $K$ such that for each $x \in K \backslash A$ there exists $y \in B$, satisfying $g(x, y)<0$.

Then there exists $\bar{x} \in K$ such that $g(\bar{x}, y) \geq 0$ for all $y \in K$.

Remark 4.2. If the bifunction $g$ in Theorem 4.2 is convex with respect to the second argument, then condition (i) of Theorem 4.2 is satisfied.

Now we can state the main result of this section.

Theorem 4.3. Let $U$ and $V$ be two nonempty and convex subsets of two Hausdorff topological vector spaces $X$ and $Y$ respectively such that $U \times V$ is a paracompact set in $X \times Y$. Let $f: U \times V \rightarrow Z$ be a vector-valued function satisfying the following conditions :

(i) for every $v \in V, f(\cdot, v)$ is $C$-properly quasiconvex and $C$-lower semicontinuous; 
(ii) for every $u \in U, f(u, \cdot)$ is $C$-properly quasiconcave and $C$-upper semicontinuous;

(iii) (Coercivity) there is a nonempty compact set $(N \times M) \subset(U \times V)$, and there is a non- empty compact convex set $(\widetilde{N} \times \widetilde{M}) \subset(U \times V)$ such that if $(x, y) \in(N \times M)^{c} \cap(U \times V)$, then $f(u, \widetilde{v})-f(\widetilde{u}, \widetilde{v}) \in-$ int $C$ and $f(\widetilde{u}, \widetilde{v})-f(\widetilde{u}, v) \in-i n t C$ for some $(\widetilde{u}, \widetilde{v}) \in \widetilde{N} \times \widetilde{M}$.

Then, problem (VSP) has at last one solution.

Proof. Let $K:=U \times V$. For $(x, y) \in K$, we consider the following sets

$$
\begin{array}{ll}
T(x, y)=\{u \in U: & \left.f(x, y)-f(u, y) \in(-i n t C)^{c}\right\} \\
L(x, y)=\{v \in V: & \left.f(x, v)-f(x, y) \in(-i n t C)^{c}\right\} .
\end{array}
$$

Note that $T(x, y) \times L(x, y)$ is nonempty since $x \in T(x, y)$ and $y \in L(x, y)$. We consider for $(x, y) \in K$ the following set

$$
W(x, y)=\{(u, v) \in N \times M: \quad u \in T(x, y) \quad \text { and } \quad v \in L(x, y)\} .
$$

We show that $\bigcap_{(x, y) \in K} W(x, y) \neq \emptyset$. Suppose, on the contrary, that this assertion is false. Then for each $(\bar{u}, \bar{v}) \in K$, there exists $(x, y) \in K$ such that $(\bar{u}, \bar{v}) \notin W(x, y)$. By the Hahn-Banach separation Theorem, there exists $p \in(X \times Y)^{*}$ such that

$$
\langle p,(\bar{u}, \bar{v})\rangle>\sup _{(w, t) \in W(x, y)}\langle p,(w, t)\rangle,
$$

that is,

$(\bar{u}, \bar{v}) \in V_{p}=\{(u, v) \in K: \exists(x, y) \in K /\langle p,(u, v)\rangle>\langle p,(w, t)\rangle, \forall(w, t) \in W(x, y)\}$.

One can easily show that $V_{p}$ is an open set. Consequently, $\left\{V_{p}\right\}_{p \in(X \times Y)^{*}}$ is an open cover of $K$. Since $K=U \times V$ is paracompact, then there is a continuous partition of unity $\left\{\beta_{p}\right\}_{p \in(X \times Y)^{*}}$ subordinated to this open cover. Consider the following real-valued function $\varphi: K \times K \rightarrow \mathbb{R}$ defined by

$$
\varphi((u, v),(w, t))=\sum_{p \in(X \times Y)^{*}} \beta_{p}(u, v)\langle p,(w, t)-(u, v)\rangle .
$$

Note that the last sum is finite because for any $\left(u_{0}, v_{0}\right) \in K$ there exist a neighborhood $\mathcal{V}\left(u_{0}, v_{0}\right)$ of $\left(u_{0}, v_{0}\right)$ and $p_{1}, \ldots, p_{n} \in(X \times Y)^{*}$ such that for all $(u, v) \in$ $\mathcal{V}\left(u_{0}, v_{0}\right)$

$$
\varphi((u, v),(w, t))=\sum_{i=1}^{n} \beta_{p_{i}}(u, v)\left\langle p_{i},(w, t)-(u, v)\right\rangle \text { for all }(w, t) \in K .
$$


We see at once that the function $\varphi$ is convex with respect to its second argument, then the condition (i) in Theorem 4.2 is satisfied. It follows easily that for all $(t, w) \in K$, the function $(u, v) \mapsto \varphi((u, v),(w, t))$ is continuous, therefore it is upper semicontinuous on $K$. Now let us set $A=N \times M$ and $B=\widetilde{N} \times \widetilde{M}$. Then $A$ is a compact subset of $K$ and $B$ is a compact convex subset of $K$.

Let $(u, v) \in A$. From assumption (iii), there exists $\left(x_{0}, y_{0}\right) \in B$ such that $u \notin$ $T\left(x_{0}, y_{0}\right)$ and $v \in L\left(x_{0}, y_{0}\right)$. Consequently $(u, v) \notin W\left(x_{0}, y_{0}\right)$. Therefore, by the Hahn-Banach separation Theorem, there exists $q \in(X \times Y)^{*}$ such that $\langle q,(w, t)-$ $(u, v)\rangle<0$ for all $(w, t) \in W\left(x_{0}, y_{0}\right)$. Let $\left\{q, p_{1}, \ldots, p_{n}\right\} \subset(X \times Y)^{*}$ be such that $\varphi((u, v),(w, t))=\beta_{q}(u, v)\langle q,(w, t)-(u, v)\rangle+\sum_{i=1}^{n} \beta_{p_{i}}(u, v)\left\langle p_{i},(w, t)-(u, v)\right\rangle$, for all $(w, t) \in W\left(x_{0}, y_{0}\right)$. Since $\left\{\beta_{p}\right\}_{p \in(X \times Y)^{*}}$ is a partition of unity, $\beta_{q}(u, v)>0$ or $\beta_{p_{i}}(u, v)>0$ for at least one index $i \in\{1, \ldots, n\}$. Consequently, $\varphi((u, v),(w, t))<$ 0 for all $(w, t) \in W\left(x_{0}, y_{0}\right)$ if $\beta_{q}(u, v)>0$. On the other hand, if $\beta_{p_{i}}(u, v)>0$ for some $i$, then from (iii) and the definition of the set $V_{p_{i}}$, there is $(x, y) \in K$ such that $\varphi((u, v),(w, t))<0$ for all $(w, t) \in W(x, y)$. Therefore, from Theorem 4.2, we deduce that there exists $(\bar{u}, \bar{v}) \in K$ such that

$$
\varphi((\bar{u}, \bar{v}),(w, t)) \geq 0 \text { for all }(w, t) \in K .
$$

Let $\left\{r_{1}, \ldots, r_{m}\right\} \subset(X \times Y)^{*}$ be such that

$$
\varphi((\bar{u}, \bar{v}),(w, t))=\sum_{i=1}^{m} \beta_{r_{i}}(\bar{u}, \bar{v})\left\langle r_{i},(w, t)-(\bar{u}, \bar{v})\right\rangle
$$

for all $(w, t) \in K$. If $\beta_{r_{i}}(\bar{u}, \bar{v})>0$ for some $i$, then $(\bar{u}, \bar{v}) \in V_{r_{i}}$. Therefore, there exists $(x, y) \in K$ such that $\left\langle r_{i},(w, t)-(\bar{u}, \bar{v})\right\rangle<0$ for all $(w, t) \in W(x, y)$ which contradicts relation (2). Consequently, the problem $(V S P)$ must have at least one solution and the result follows.

\section{CONCLUSion}

In this paper, we have studied the existence of solutions of a vector saddlepoint problem by different approaches. More precisely, we derive existence results by means of an extension to the vector setting of a Brezis-Nirenberg-Stampacchia condition, which leads us to relax the $C$-lower (upper) semicontinuity assumption due to T.Tanaka when dealing with vector saddle-point problems. In a second approach, we present a treatment of vector saddle-point problems by means of a paracompacity assumption. We argue that a similar treatment can be considered for the following vector saddle-point problems considered in the literature: 


$$
\begin{aligned}
& (V S P)_{1}\left\{\begin{array}{l}
\text { find a pair }\left(u_{0}, v_{0}\right) \in U \times V \text { such that } \\
f\left(u, v_{0}\right)-f\left(u_{0}, v_{0}\right) \in C \forall u \in U \text { and } \\
f\left(u_{0}, v_{0}\right)-f\left(u_{0}, v\right) \notin-i n t C \forall v \in V,
\end{array}\right. \\
& (V S P)_{2}\left\{\begin{array}{l}
\text { find a pair }\left(u_{0}, v_{0}\right) \in U \times V \text { such that } \\
f\left(u, v_{0}\right)-f\left(u_{0}, v_{0}\right) \in C \forall u \in U \text { and } \\
f\left(u_{0}, v_{0}\right)-f\left(u_{0}, v\right) \in C \forall v \in V,
\end{array}\right. \\
& (V S P)_{3}\left\{\begin{array}{l}
\text { find a pair }\left(u_{0}, v_{0}\right) \in U \times V \text { such that } \\
f\left(u, v_{0}\right)-f\left(u_{0}, v_{0}\right) \in C \forall u \in U \text { and } \\
f\left(u_{0}, v_{0}\right)-f\left(u_{0}, v\right) \in C \forall v \in V .
\end{array}\right.
\end{aligned}
$$

\section{REFERENCES}

1. K. L. Chew, Maximal points with respect to cone dominance in banach spaces and their existence, J. Optim. Theory Appl., 44 (1984), 1-53.

2. P. L. Yu, Cone convexity, cone extreme points, and nondominated solutions in decision problems with multiobjectives, J. Optim. Theory Appl., 14 (1974), 319-377.

3. F. Ferro, A minimax theorem for vector-valued functions, J. Optim. Theory Appl., 60(1) (1989), 19-31.

4. K. R. Kazmi and S. Khan, Existence of solutions for a vector saddle point problem, Bull. Austral. Math. Soc., 61 (2000), 201-206.

5. K. Kimura, Existence results for cone saddle points by using Vector-variational-like inequalities, Nihonkai Math. J., 15(1) (2004), 23-32.

6. K. Kimura and T. Tanaka, Existence theorem of cone saddle-points applying a nonlinear scalarization, Taiwanese J. Math., 10(2) (2006), 563-571.

7. D. T. Luc, Theory of Vector Optimization, Lecture Notes in Economics and Mathematical Systems, 319, Springer-Verlag, Berlin, 1989.

8. K. Kimura, On some types of vectorial saddle-point problems, Nihonkai Mathematical Journal, to appear.

9. W. Takahashi, Nonlinear Functional Analysis-Fixed Point Theory and its Applications, Yokohama-Publishers Yokohama, 2000.

10. P. Deguire, K. K. Tan and G. X.-Z. Yuan, The study of maximal elements, fixed points for $L_{S}$-majorized mappings and their applications to minimax and variational inequalities in product topological spaces, Nonlinear Analysis, 37(7) (1999), 933-951.

11. T. Tanaka, Generalized semicontinuity and existence theorems for cone saddle points, Appl. Math. Optim., 36 (1997), 313-322. 
12. T. Tanaka, Cone-quasiconvexity of vector-valued functions, Sci. Rep. Hirosaki Univ., 42 (1995), 157-163.

13. T. Tanaka, Generalized quasiconvexities, cone saddle points, and minimax theorem for vector-valued funtions, J. Optim. Theory Appl., 81 (1994), 355-357.

14. K. Fan, A generalization of Tychonoff's fixed point theorem, Math. Ann., 142 (1961), 305-310.

15. H. Brézis, L. Nirenberg and G. Stampacchia, A ramark on Ky Fan's minmax principale, Boll. Un. Mat. Ital., 4 (1972), 293-300.

16. H. Brézis, Equations et inéquations non Linéaires dans les espaces vectoriels en Dualité, Ann. Inst. Fourier Grenoble, 18 (1968), 123-145.

17. O. Chadli, Y. Chiang and S. Huang, Topological pseudomonotonicity and vectorvalued equilibrium problems, J. Math. Anal. Appl., 270 (2002), 435-450.

18. Q. H. Ansari and J. C. Yao, An existence result for the generalized vector equilibrium problem, Appl. Math. Lett., 128 (1999), 53-56.

19. O. Chadli, X. Q. Yang and J. C. Yao, On generalized vector pre-variational and pre-quasivariational inequalities, J. Math. Anal. Appl., 295 (2004), 392-403.

20. G. Chen, X. Huang and X. Q. Yang, Vector Optimization: Set-valued and Variational Analysis, Lecture Notes in Economics and Mathematical Systems, 2005.

21. J. P. Aubin, Mathematical Methods of Game and Economic Theory, Studies in Mathematics and its Applications, Vol. 7, North Holland, 1979.

22. C. D. Aliprantis and K. C. Border, Infinite Dimensional Analysis: A Hitchhiker's Guide, 3rd. ed. Berlin, Springer-Verlag, 2006.

23. C. Zalinescu, Convex Analysis in General Vector Spaces, World Scientific, 2002.

24. O. Chadli, Z. Chbani and H. Riahi, Equilibrium problems with generalized monotone bifunctions and applications to variational inequalities, J. Optim. Theory Appl., 105 (2000), 299-323.

O. Chadli

Department of Economics

Faculty of Economics and Social Sciences

Ibn Zohr University

Agadir, Morocco

E-mail: ochadli@esta.ac.ma

H. Mahdioui

Department of Mathematics

Faculty of Sciences

Ibn Zohr University

Agadir, Morocco 